\author{
Stefano SCALERCIO* - Salvatore UrSO** - Silvia GRECO* - Marco INfUSINO*
}

\title{
Alcune specie di Pyraloidea nuove per la fauna dell'Italia meridionale (Lepidoptera, Pyralidae, Crambidae)
}

\begin{abstract}
Riassunto: In questo lavoro sono riportati dati di cattura che ampliano significativamente la distribuzione italiana di sei specie di Pyraloidea. Acrobasis bithynella e Sciota rhenella sono segnalate per la prima volta in Italia centro-meridonale, mentre Pempelia alpigenella e Peoria cremoricosta sono segnalate per la prima volta in Italia meridionale peninsulare. Inoltre, per Hypsotropa vulneratella e Hyperlais argillacealis si tratta dei primi reperti per la penisola di specie note finora in Italia solo in Sicilia.
\end{abstract}

\begin{abstract}
Some Pyraloidea new to the fauna of forested ecosystems of Southern Italy (Lepidoptera, Pyralidae, Crambidae).
In this paper sampling data concerning six species belonging to the Pyraloidea are reported, significantly improving the knowledge on their presence in Italy. Acrobasis bithynella and Sciota rhenella were so far known only for northern Italy, while Pempelia alpigenella and Peoria cremoricosta were reported for the second time in Italy and for the first time in southern regions. Furthermore, Hypsotropa vulneratella and Hyperlais argillacealis were so far known only for Sicily.
\end{abstract}

Key words: Biodiversity, Calabria, Cybalomiinae, Distribution, Phycitinae.

\section{INTRODUZIONE}

Negli ultimi anni la fauna italiana dei Pyraloidea viene continuamente aggiornata con segnalazioni di specie nuove o poco conosciute per l'Italia (Pinzari et al., 2010; Baldizzone et al., 2013; Scalercio et al., 2014; Zilli \& Pavesi, 2015; Scalercio, in stampa). L'Italia meridionale, finora esplorata in maniera piuttosto occasionale, ha fornito molte delle novità rinvenute, vedendo significativamente incrementate le conoscenze faunistiche disponibili. Lo scopo di questo lavoro è di contribuire a delineare la fauna dei Pyraloidea della Calabria, una regione per la quale sono disponibili solo dati isolati. L'attenzione è stata rivolta in particolare agli ecosistemi forestali, i quali dominano il paesaggio della regione.

\section{MATERIALI E METODI}

Il materiale è stato raccolto utilizzando diversi metodi, ricordati di volta in volta nella elencazione delle specie. Salvo diversa indicazione, i dati di presenza in Europa sono ripresi dalla Fauna Europaea (Karsholt \& Nieukerken, 2013), mentre la distribuzione italiana è ripresa da Bassi et al. (1995). Le specie sono state identificate utilizzando la bibliografia di- sponibile (Roesler, 1973, 1993; Parenti, 2000; Slamka, 2010; Ylla et al., 2012).

Di seguito si riporta l'elenco in ordine alfabetico delle località citate e una loro descrizione di massima:

- Belvedere di Malvento, Morano Calabro (CS), Pollino-Orsomarso, $1580 \mathrm{~m}$, latitudine: $39,9025^{\circ}$, longitudine: $16,1372^{\circ}$, radura xerica in faggeta;

- Bosco Gesuiti, San Fili (CS), Catena Costiera, 530 $\mathrm{m}$, latitudine: $39,3620^{\circ}$, longitudine: $16,1309^{\circ}$, castagneto;

- Capo Spartivento, Palizzi Marina (RC), Costa Ionica, $3 \mathrm{~m}$, latitudine: $37,9193^{\circ}$, longitudine: $16,006^{\circ}$, retroduna costiero.

- Contrada Li Rocchi, Rende (CS), Valle Crati, 210 $\mathrm{m}$, latitudine: $39,3689^{\circ}$, longitudine: $16,2286^{\circ}$, area ruderale suburbana;

- Fiego di San Fili, San Fili (CS), Catena Costiera, $720 \mathrm{~m}$, latitudine: $39,3372^{\circ}$, longitudine: $16,129^{\circ}$, castagneto;

- Fosso Cucolo, Donnici Inferiore (CS), Sila, $550 \mathrm{~m}$, latitudine: $39,2369^{\circ}$, longitudine: $16,2972^{\circ}$, margine di querceto di Quercus pubescens Willd.;

- Glicarello, Montalto Uffugo (CS), Catena Co-

\footnotetext{
*Stefano Scalercio, Silvia Greco, Marco Infusino, Consiglio per la ricerca in agricoltura e l'analisi dell'economia agraria, Unità di Ricerca per la Selvicoltura in Ambiente Mediterraneo, Contrada Li Rocchi, 87036 Rende (CS), Italia. E-mail: stefano.scalercio@crea.gov.it,marco.infusino@crea.gov.it, silvia.greco@crea.gov.it

${ }^{* *}$ Salvatore Urso, Associazione TartaCare ONLUS, Via G. Gronchi 6, 87100 Cosenza, Italia. E-mail: salvourso78@gmail.com
} 
stiera, $550 \mathrm{~m}$, latitudine: $39,4072^{\circ}$, longitudine: $16,1256^{\circ}$, castagneto;

- Montagna Grande, San Giovanni in Fiore (CS), Sila, $1355 \mathrm{~m}$, latitudine: $39,2717^{\circ}$, longitudine: $16,6062^{\circ}$, pineta a Pinus laricio Poiret;

- Vallone Argentino, Montalto Uffugo (CS), Catena Costiera, $565 \mathrm{~m}$, latitudine: $39,4082^{\circ}$, longitudine: $16,1209^{\circ}$, castagneto.

\section{ELENCO DELLE SPECIE}

Familia Pyralidae Latreille, 1809

Subfamilia Phycitinae Zeller, 1839

Tribus Phycitini Zeller, 1839

\section{Acrobasis bithynella Zeller, 1848 (Fig. 1)}

REPERTI. Contrada Li Rocchi, 01.X.2014, 10̂; idem, 10, 31.X.2014; Fosso Cucolo, 12.X.2014, $10^{\Uparrow}$.

OSSERVAZIONI. Raccolta vicino alle illuminazioni esterne di fabbricati, rappresentate da lampade ad incandescenza da 100W. La larva si alimenta di diverse specie del genere Cistus (Lhomme, 1935; Huertas Dionisio, 2009) e Quercus (Parenti, 2000).

DistribuZione nOTA. Nota in Europa per Corsica, Creta, Croazia, Francia, Italia, Portogallo, Russia, Sardegna, Sicilia, Spagna e Turchia. In Italia continentale è conosciuta solo per le regioni settentrionali.

Nuova per l'Italia centro-meridionale.

Pempelia alpigenella (Duponchel, 1836) (Fig. 2) REPERTI. Belvedere di Malvento, 27.VII.2014, 1 万. OSSERVAZIONI. Raccolta manualmente durante le ore diurne. Non abbiamo informazioni circa la biologia larvale.

DistriBuZIONE NOTA. Nota in Europa per Albania, Bulgaria, Francia, Grecia, Italia, Macedonia, Russia e Ucraina. In Italia è segnalata per l'Abruzzo (Bassi et al., 1995) e recentemente per il Lazio (Pinzari et al., 2010).

Nuova per l'Italia meridionale.

Sciota rhenella (Zincken, 1818) (Fig. 3)

REPERTI. Glicarello, 22.VII.2015, 10'; Vallone Argentino, 22.VII.2015, 1ठ; Bosco Gesuiti, 22.VII.2015, 1 ㅇ. OsSERVAZIONI. Rinvenuta in trappole luminose tipo "Heath" aventi come fonte luminosa LED ad UV. La larva si alimenta di alcune specie di Salix e Populus (Roüast, 1884).

DistRIBUZIONE NOTA. Nota in Europa per Austria, Bel- gio, Bulgaria, Danimarca, Estonia, Finlandia, Francia, Germania, Italia, Lettonia, Lituania, Olanda, Polonia, Repubblica Ceca, Romania, Russia, Slovacchia, Spagna, Svizzera, Ungheria. Nota in Italia solo al settentrione e in Toscana (Palm, 2012).

Nuova per l'Italia meridionale.

Tribus Anerastiini Ragonot, 1885

Hypsotropa vulneratella (Zeller, 1847) (Fig. 4)

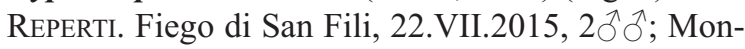
tagna Grande, 17.VII.2015, $1 \delta^{\Uparrow}$.

OSSERVAZIONI. Rinvenuta in trappole luminose tipo "Heath" aventi come fonte luminosa LED ad UV. Non abbiamo informazioni circa la biologia larvale.

DistRIBUZIONE NOTA. Nota in Europa per Croazia, Francia, Italia, Malta, Portogallo e Spagna. In Italia è conosciuta per Sardegna e Sicilia.

Nuova per l'Italia continentale.

Peoria cremoricosta (Ragonot, 1895) (Fig. 5)

RePERTI. Capo Spartivento, 15.V.2015, $2{ }^{\top}{ }^{\top}$.

OSSERVAZIONI. Raccolta con una trappola luminosa tipo "Heath" avente come fonte luminosa una lampada a luce miscelata da $160 \mathrm{~W}$. Trasferita nel genere Peoria da Leraut (2014). Riportata in Fauna Europaea (Karsholt \& Nieukerken, 2013) come Seleucia pectinella (Chrétien, 1911) che in realtà è sinonimo di Peoria cremoricosta (Ylla et al., 2012). Non abbiamo informazioni circa la biologia larvale.

Distribuzione nota. Conosciuta in Europa per Penisola Iberica, Albania, Creta, Croazia, Grecia e Italia, dove è nota solo per Sicilia come S. pectinella (Bassi et al., 1995) e Lazio come Fregenia prolai (Hartig, 1947). Nuova per l'Italia meridionale.

Familia Crambidae Latreille, 1810

Subfamilia Cybalomiinae Marion, 1955

Hyperlais argillacealis (Zeller, 1847) (Fig. 6) REPERTI. Capo Spartivento, 15.V.2015, 1 , $10^{\Uparrow}$. OSSERVAZIONI. Raccolta con una trappola luminosa tipo "Heath" avente come fonte luminosa una lampada a luce miscelata da $160 \mathrm{~W}$. Non abbiamo informazioni circa la biologia larvale.

DisTRIBUZIONE NOTA. Nota in Europa per Creta, Croazia, Grecia, Italia e Macedonia. In Italia è conosciuta solo per la Sicilia.

Nuova per l'Italia continentale. 


\section{RINGRAZIAMENTI}

Ringraziamo Carlo di Marco, Enzo Calabrese, Gino Scarpelli, Massimo ed Ettore Salerno per l'aiuto su campo ed il supporto tecnico. Ringraziamo inoltre gli enti del Parco Nazionale del Pollino e del Parco Nazionale della Sila e i proprietari terrieri che ci hanno rilasciato i permessi del caso. La ricerca è stata parzialmente finanziata dal Progetto "ALForLab" (PON03PE_00024_1) cofinanziato dal Programma Operativo Nazionale per la Ricerca e la Competitività (PON R\&C) 2007-2013, attraverso l'European Regional Development Fund (ERDF) e risorse nazionali [Revolving Fund - Cohesion Action Plan (CAP) MIUR].
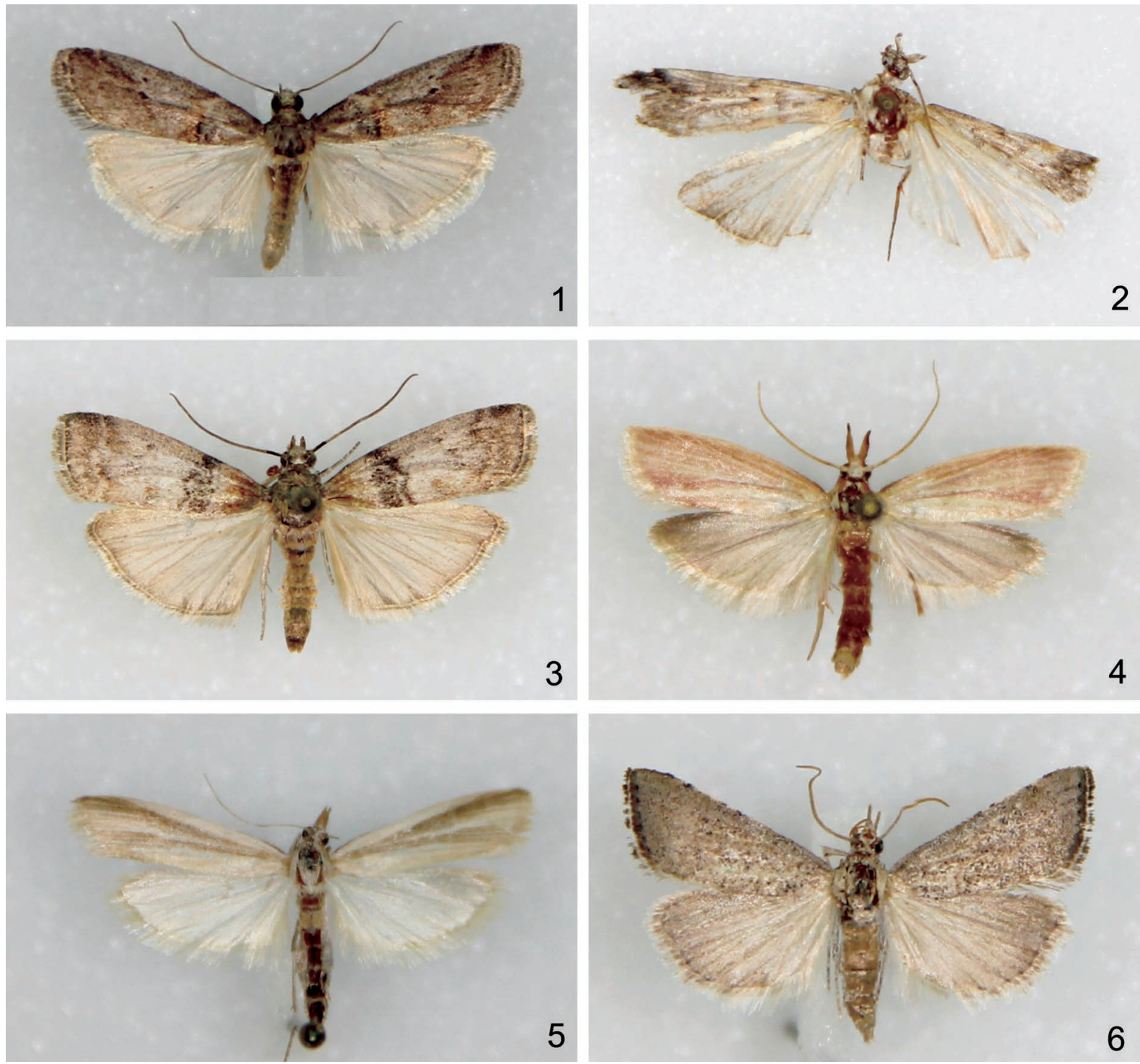

Figg. 1-6. 1 - Acrobasis bithynella, Contrada Li Rocchi, 01.X.2014, ô; 2 - Pempelia alpigenella, Belvedere di Malvento, 27.VII.2014, ô; 3 - Sciota rhenella, Glicarello, 22.VII.2015, ô; 4 - Hypsotropa vulneratella, Fiego di San Fili, 22.VII.2015,

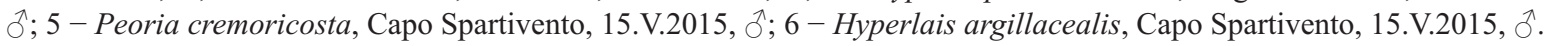




\section{BIBLIOGRAFIA}

Baldizzone G., Cabella C., Fiori F., Varalda P.G., 2013 - I Lepidotteri del Parco Naturale di Marcarolo. Memorie dell’Associazione Naturalistica Piemontese, XII: 349 pp.

Bassi G., Passerin D’Entreves P., Speidel W., Zangheri S., 1995 - Lepidoptera Pyraloidea. In: Minelli A., Ruffo S., La Posta S. (eds.). Checklist delle specie della fauna italiana, 87. Calderini, Bologna.

Hartig F., 1947 - Un nuovo genere di Anerastiidae (Lepid., Pyralid.) del Lazio. Fregenia n. g. Bollettino dell'Associazione Romana di Entomologia 2 (4): 31-32.

Huertas Dionisio M., 2009 - Estados inmaturos de Lepidoptera (XXXV). Seis especies y dos subespecies del género Acrobasis Zeller, 1839 en Huelva, España (Lepidoptera: Pyralidae, Phycitinae). SHILAP Revista de Lepidopterología, 37 (145): 65-99.

Karsholt O., Van Nieukerken E.J., 2013 - Fauna Europaea: Lepidoptera, Moths. Fauna Europaea version 2.6.2, http://www.faunaeur.org.

Lhomme L., 1935 - Catalogue des lépidoptères de France et de Belgique. Microlépidoptères, 2: 1-172. Le Carriol, Par Douelle, Lot.

PALM E., 2012 - Rare or rarely pictured species of Phycitidae (Pyralidae). Part 1. Lepidoptera - Medlemsblad for Lepidopterologisk Forening, Copenhagen 10 (3): 97-114.

Parenti U., 2000 - A guide to the Microlepidoptera of Europe. Guide I. 2000. Museo Regionale di Scienze Naturali Torino, $426 \mathrm{pp}$.

PinZARi M., PinZARi M., ZiLli A., 2010 - Deep lepidopterological exploration of Mt Cagno and surroundings (Central Italy), a restricted mountain massif and hotspot for butterfly and moth diversity. Bollettino dell'Associazione Romana di Entomologia, 65 (1-4): 3-383.

Roesler R.U., 1973 - Phycitinae. Trifine Acrobassiina. In: Amsel H.G., Gregor F., Reisser H. (eds.), Microlepidoptera Palaearctica 4. Vol. 1. \& 2. Georg Fromme \& Co., Wien, i-xvi, 752 pp. \& 137 pp., pls. 1-170.

Roesler R. U., 1993 - Quadrifine Acrobasiina. 2. Teilband der Phycitinae. In: Amsel H.G., Gregor F., Reisser H., Roesler R.U. (eds.). Microlepidoptera Palaearctica, 8: XXII + 305 pp.,82 pls. G. Braun, Karlsruhe.

RoüAst G., 1884 - Catalogue des chenilles Européennes connues. Annales de la Société Linnéenne de Lyon, Nouvelle Série, 30 (1883): 70-152.

SCALERCIO S., in press - Interessanti novità faunistiche sui Pyraloidea dell'Italia meridionale con particolare riferimento agli ambienti forestali (Lepidoptera: Pyralidae, Crambidae). SHILAP Revista de Lepidopterología.

SCAlERCio S., LuZZi G., Infusino M., 2014 - First record of Pempelia amoenella (Zeller, 1848) for Western Europe (Lepidoptera Pyralidae). Biodiversity Journal, 5(2): 217-220.

Slamka F., 2010 - Pyraloidea (Lepidoptera) of Central Europe. 3. Edition. Slamka, Bratislava, 176 pp.

Ylla, J., Zilli, A., Macià R., 2012 - Polyocha cremoricosta Ragonot, 1895 en la Península Ibérica (Lepidoptera: Pyralidae, Phycitinae, Anerastiini). SHILAP Revista de Lepidopterología 40 (159): 299-310.

Zilli A., PAVesi F., 2015 - New or little known Pyraloids from Italy (Lepidoptera: Pyraloidea). Phegea, 43 (3): 81-96. 